\title{
Sensitivity and Coding of Opportunistic ALOHA in Sensor Networks with Mobile Access
}

\author{
PARVATHINATHAN VENKITASUBRAMANIAM \\ Department of Electrical and Computer Engineering, Cornell University, Ithaca, NY 14853, USA \\ SRIHARI ADIREDDY \\ Silicon Laboratories, Austin, Texas \\ LANG TONG \\ Department of Electrical and Computer Engineering, Cornell University, Ithaca, NY 14853, USA
}

\begin{abstract}
We consider a distributed medium access protocol, Opportunistic ALOHA, for reachback in sensor networks with mobile access points (AP). We briefly discuss some properties of the protocol, like throughput and transmission control for an orthogonal CDMA physical layer. We then consider the incorporation of necessary side information like location into the transmission control and numerically demonstrate the loss in throughput in the absence of such information. Through simulations, we discuss the robustness and sensitivity of the protocol under various modeling errors and propose strategies to allow for errors in estimation of some parameters without reduction in the throughput. For networks, where the sensors are allowed to collaborate, we consider three coding schemes for reliable transmission: spreading code independent, spreading code dependent transmission and coding across sensors. These schemes are compared in terms of achievable rates and random coding error exponents. The coding across sensors scheme has comparable achievable rates to the spreading code dependent scheme, but requires the additional transmission of sensor ID. However, the scheme does not require the mobile AP to send data through the beacon unlike the other two schemes. The use of these coding schemes to overcome sensitivity is demonstrated through simulations.
\end{abstract}

Keywords: sensor networks, mobile access point, sensitivity, coded random access

\section{Introduction}

Medium access control (MAC) is crucial in large scale sensor networks. Some of the most difficult challenges include the presence of a large number of potentially interfering sensors, the lack of synchronization among sensors, the lack of feedback in some cases, and severe power constraints on transmission power. These special features of sensor networks call for a reex- amination of MAC strategies. Furthermore, the possibility of sensors collaboratively transmitting messages brings a new dimension in MAC design and challenges the traditional view of layered network design.

MAC design is intimately connected with the network architecture. We consider SENMA-Sensor Networks with Mobile Access-for large scale wireless sensor networks [13, 14, 25]. As shown in Fig. 1, 


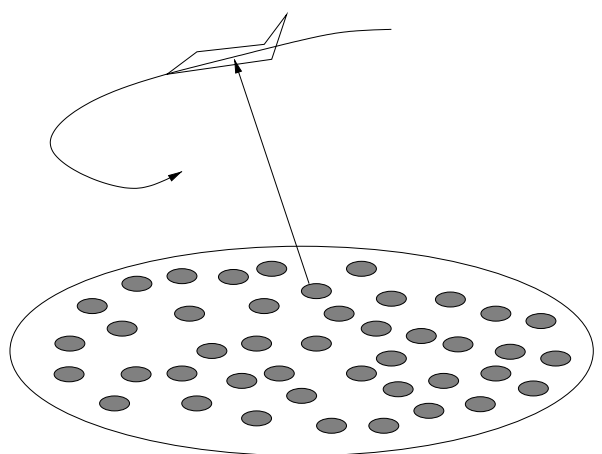

Figure 1. Sensor network with mobile access point.

SENMA has two types of nodes: a large number of low power sensors distributed randomly on a field and a few more powerful mobile access points (APs). The mobile access points can be unmanned ground/aerial vehicles that move randomly around the field equipped with sophisticated receivers and processors and may have connection to satellites for reachback to remote control centers. Similar architectures have been proposed by many researchers for different applications $[12,15,22,23,26]$.

A fundamental difference between SENMA and Ad hoc sensor networks is that, in SENMA, sensors communicate not directly among themselves but through mobile APs. This shifts many network functions and management away from the sensor network to a small set of power nodes. Another key characteristic of SENMA is the mobility of the access points. Mobility introduces diversity and improves overall network throughput [11]. In the context of sensor networks, the mobility of the APs makes it possible for sensors to exploit channel state information.

We consider a distributed random access protocol Opportunistic ALOHA (O-ALOHA) for retrieving information from sensors. The idea of O-ALOHA, first considered in [10,21], is to let each sensor transmit based on its own channel state; a sensor transmits with high probability if it has a good channel to the mobile AP and avoids transmission when the channel is poor. The benefit of such opportunistic transmissions is twofold. First, it enhances the chance that the transmission will be successful thereby improving the throughput of the network. Second, it avoids unnecessary transmissions that have little chance to succeed and create only interference.

\subsection{Related Work}

Previously, several different aspects of sensor networks have been considered under the SENMA architecture. Distributed detection with a large number of identical sensors was considered in [9, 20]. In [8, 19], the authors focus on the impact of MAC design on the reconstruction performance of a random signal field. Energy-efficient distributed MAC schemes for SENMA were proposed in [18]. In [16, 17], the authors discuss optimal configurations of multiple mobile APs for maximizing throughput and efficiency. The hierarchical architecture for sensor networks, where mobile access points perform most of the network functions has been considered in different forms [12, 15, 22, $23,26]$. There has also been a lot of work in the recent past on medium access in ad-hoc sensor networks $[4,7]$. Self-Organizing medium access for sensor networks or SMACS [12] is a distributed protocol a collection of sensors discover their neighbours and schedule communications. They however do not require the use of mobile nodes. The EAR algorithm (Eavesdrop and Register) [12] coniders medium access for sensor networks where mobile nodes are incorporated into the system. These mobile nodes maintain a partial registry, initiate hand-shaking when required and are not responsible for data transfer. The use of mobile nodes called message ferries to carry messages between stationary disconnected nodes was proposed in [15]. The main consideration here is proactive routing by exploiting non-randomness in the mobility of the message ferries. In [26], mobile entities called MULEs pick up data from the sensors in close range and drop it off at access points.

\subsection{Main Results and Organization}

This paper focuses on several new aspects of OALOHA not considered in the past [13, 14, 25]. In particular, we are interested in the sensitivity of O-ALOHA under various modeling errors. A brief description of the protocol is given in Section 2. In Section 4.1, we discuss two methods of incorporating side information like location into the protocol, and plot the variation of throughput in the absence of such information. The throughput is found to decrease when necessary side information is not available. In Sections 5.1, 5.2, 5.3, and 5.4 we look at the sensitivity of O-ALOHA under errors in the path-loss parameter, rayleigh fading parameter, channel gain and size 
of the network respectively. The protocol is found to be sensitive to errors in fading and path-loss parameters, primarily due to the exponential dependence on these parameters. It is however fairly robust to changes in network size and error in estimated channel gain. When the error in channel gain is due to an additive noise, we show that the transmission control can be modified to handle the error and achieve the desired asymptotic throughput.

In Section 6, we discuss three schemes that combine coding and random access for reliable transmission. The first two schemes-Spreading Code Independent Transmission and Spreading Code Dependent Transmission-require the sensors to decode information from the beacon. These schemes were previously discussed in [13]. The new scheme proposed in this paper-Coding Across Sensors-does not require decoding of beacons, but mandates the transmission of node ID in every packet. We show that the achievable rate for the spreading code dependent and coding across sensors scheme are higher whereas for low rate codebooks, the spreading code independent scheme has a better error exponent. In Section 7.3, we demonstrate how coding can be used to overcome the sensitivity of the protocol to fading parameters. Conclusions are given in Section 8.

\section{Opportunistic ALOHA}

\subsection{Protocol Discipline}

In this section, we briefly describe the working of the O-ALOHA protocol [10, 21]. We consider a sensor network, where $n$ sensors try to communicate to a mobile access point (AP) over a common time-slotted channel.

The slot structure is shown in Fig. 2. All slots have identical lengths equal to the duration of transmission of one packet. The network is assumed to operate in the time division duplex (TDD) mode. The mobile AP broadcasts a beacon at the beginning of each slot, which is used by the sensors to estimate the channel gain from the mobile AP to itself. The beacon is also used by the sensors for time synchronization. Due to reciprocity, the channel gain between the $i$ th sensor and the mobile

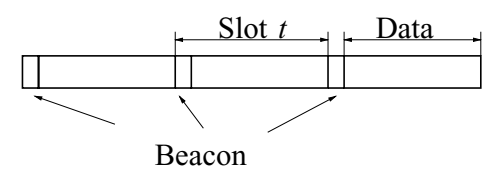

Figure 2. Slot structure.
AP during slot $t$, denoted by $\gamma_{i}{ }^{(t)}$, is assumed to be the same as the channel gain estimated from the beacon at the beginning of that slot. Each sensor then transmits its packet with a probability $\mathrm{s}\left(\gamma_{i}{ }^{(t)}\right)$, known as the transmission control. The transmission control will be discussed in more detail in Section 3.2.

\subsection{Data Transmission and Reception}

The physical layer of the sensor network is based on orthogonal CDMA with spreading gain $N$. There is a pool of $N$ orthogonal codes, and each transmitting sensor randomly selects one of these $N$ codes to transmit its data. The receiver at the mobile AP uses $N$ matched filters to demodulate the received data. We ignore the interference from users transmitting on other orthogonal spreading codes. Therefore, it is enough to consider the output of a single matched filter. We assume that a packet is received successfully if the signal to interference-noise ratio (SINR) is greater than a threshold $\beta$ [6]. Specifically, in slot $t$, if $k$ sensors choose to transmit using the same orthogonal code, and their channel gains are given by $\left(\gamma_{1}, \ldots, \gamma_{k}\right)$, the $i$ th sensor is successful if

$$
\frac{P_{T} \gamma_{i}}{\sigma^{2}+\sum_{j=1, j \neq i}^{k} P_{T} \gamma_{j}}>\beta,
$$

where $P_{T}$ is the transmitting power and $\sigma^{2}$ the variance of the background noise. The parameter $\beta$ is the minimum SINR needed for successful reception and is determined by factors such as type of modulation and receiver sensitivity. Depending on the value of $\beta$, multiple packet reception is possible per orthogonal code. However, if we assume $\beta>1$, then at most one packet can be captured per orthogonal code [6]. Therefore, the maximum throughput possible per slot will be equal to the spreading gain $N$.

\section{Properties of Opportunistic ALOHA}

\subsection{Throughput}

We consider the throughput to be the number of packets successfully decoded per slot, as defined in [13]. This is a function of the underlying channel state CDF $F(\gamma)$, network size $n$, reception model and the transmission control $s(\gamma)$. It is assumed that the distribution of channel state is i.i.d across sensors and from slot to slot. It has been shown [10] that the probability 
of successful reception is dependent on the aposteriori (as seen by the receiver) channel state distribution

$$
T(\gamma)=\frac{1}{p_{s}} \int_{0}^{\gamma} s d F
$$

where

$$
p_{s}=\int s d F \neq 0
$$

is the unconditional probability of transmission. The throughput per slot (per spreading code) is given by

$$
\lambda_{n}(s(\cdot))=\sum_{k=1}^{n}\left(\begin{array}{l}
n \\
k
\end{array}\right)\left(1-q p_{s}\right)^{n-k}\left(q p_{s}\right)^{k} C_{k}(T(\cdot)),
$$

where $q$ is the probability of choosing a particular spreading code conditioned on a sensor transmitting. $C_{k}(T(\cdot))$ is the average number of packets successfully received when $k$ users transmit and their channel states are drawn from the distribution $T(\cdot)$. It is clear that the throughput is dependent on the aposteriori channel state distribution $\mathrm{T}(\cdot)$. Therefore, it is possible to obtain a high throughput by manipulating the channel state distribution through the transmission control.

We are interested in the performance of very large sensor networks. Therefore, we use the notion of the asymptotic throughput of a network. As in $[10,13]$, the asymptotic throughput (for systems with $n \rightarrow \infty$ ) can be defined as

$$
\lambda_{\infty}(s(\cdot)) \triangleq \lim _{n \rightarrow \infty} \lambda_{n}(s(\cdot)) .
$$

For large networks, it is possible to obtain good asymptotic behaviour based on this metric.

\subsection{Transmission Control}

In this section, we consider a general form of transmission control that demonstrate good performance for the physical layer under consideration. The fundamental approach is to modify the aposteriori distribution in order to increase the throughput. The transmission control can also be used to control interference by regulating the average number of transmitting sensors.

Let the underlying channel state be distributed according to $F(\cdot)$. Let $T(\cdot)$ be a distribution function that is absolutely continuous with respect to $F(\cdot)$ i.e., $T(\cdot) \ll$
$F(\cdot)$. We choose the sequence of transmission controls as

$$
s_{n}(\gamma)=q \min \left(\frac{x}{n} \frac{d T}{d F}, 1\right),
$$

where we incorporate $q$-the probability of choosing a spreading code-into the transmission control $s_{n}(\gamma)$. The parameter $x$ represents the average number of transmissions per slot per orthogonal code. For such a transmission control, the asymptotic throughput has been characterized in $[10,21]$ and is given by

$$
\lambda_{\infty}\left(x, s_{n}(\cdot)\right)=e^{-x q} \sum_{k=1}^{\infty} \frac{(x q)^{k}}{k !} C_{k}(T(\cdot))
$$

The above throughput can be optimized by choosing an appropriate target distribution. For the physical layer in consideration, it was shown that good target pdf are distributions with a roll-off $[10,13]$. The choice of target distribution was motivated by [6], where the authors show that when the channel state distribution has a roll-off, the probability of capture is non-zero even when the number of transmitting users is very large. Any pdf that is of the form

$$
g(\gamma)=\frac{\delta}{\gamma_{0}^{-\delta}} \frac{1}{\gamma^{1+\delta}} 1_{\left[\gamma_{0}<\gamma\right]},
$$

where $0<\delta<1$, is considered to be a density function with roll-off. The parameters $\gamma_{0}$ and $\delta$ have to be optimized in order to maximize the throughput. It is shown in [13] that by choosing the parameters appropriately, the transmission control achieves the maximum possible throughput, equal to the spreading gain $N$.

\section{Incorporating Side Information}

In some applications, additional side information maybe necessary to determine the exact channel state distribution. For example, the distance between the sensor and the mobile AP can influence the attenuation or path loss. Such location information can be computed via the use of geolocation devices. In the absence of such devices, it is also possible to obtain the distribution of the location information from the distribution of sensors around the field and the mobility pattern of the collecting agent. Such side information can therefore be incorporated into the transmission control using two methods: one with the realization of 
the side information, the other using only the distribution. The absence of any such information however results in an erroneous assumption of prior distribution and hence the aposteriori distribution does not converge to the designed target distribution. We shall now briefly describe the two methods with location as the side information.

\subsection{Location Independent and Location Aware Transmission Controls}

By Location Independent Transmission Control (LIT), we mean that the transmission control is not dependent on the exact location, but only the distribution of the location parameter. In other words, if the location information $r$ has a pdf $g(\cdot)$, then the transmission control is only a function of $g(\cdot)$. Assuming that the pdf of the channel state conditioned on the location $r$ is given by $f(\gamma \mid \mathrm{r})$, the LIT control can be written as

$$
s_{n}(\gamma)=q \min \left(\frac{t(\gamma)}{\int f(\gamma \mid r) g(r) d r} \frac{x}{n}, 1\right)
$$

Implementation of the LIT control does not require use of geolocation devices, and can be done apriori to the sensor deployment.

By Location Aware Transmission (LAT) control we mean that the sensor has access to the location parameter $r$. The transmission probability is directly dependent on the parameter. If the pdf of the channel state conditioned on $r$ is given by $f(\gamma \mid r)$, the LAT control can be written as

$$
s_{n}(\gamma, r)=q \min \left(\frac{t(\gamma)}{f(\gamma \mid r)} \frac{x}{n}, 1\right)
$$

Since the transmission control of each sensor is different and dependent on their respective locations, LAT is more complicated to implement and would demand more processing on the part of the sensors. However, the LAT control ensures a uniform distribution of transmitting and successful sensors over the field, whereas the LIT control results in the reduction of density of transmitting and successful sensors with increase in distance from mobile AP [13].

\subsection{Numerical Results}

We now compare the performance of the protocol in the presence and absence of location information.

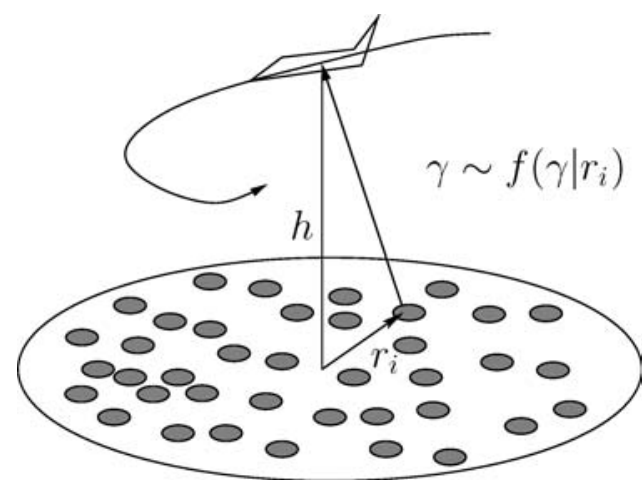

Figure 3. Sensor deployment.

We consider a practical Rayleigh fading channel with path-loss dependent on the distance between the sensor and mobile AP. We assume that all the sensors are uniformly located in a disc of radius 1 . As shown in Fig. 3, the mobile AP is assumed to be at a distance $h=1$ above the center of the disc. Let $r_{i}$, the radial distance of sensor $i$, be modeled as a random variable that has a pdf $g(r)=2 r, 0<r<1$. The transmit SNR is assumed to be $6 \mathrm{~dB}$. The propagation channel gain between sensor $i$ and the base station is modeled as

$$
\gamma_{i}^{(t)}=\frac{R_{i t}^{2}}{r_{i}^{2}+h^{2}}
$$

where $R_{i t}$ is Rayleigh distributed. We assume that $R_{i t}$ are independent and identically distributed across sensors and time. The attenuation due to distance is based on a free-space path loss model [1]. Let $f(\gamma)$ be the probability density function (pdf) of $\gamma i^{(t)}$. We also use $f(\gamma \mid r)$ to denote the probability density function of the channel state given the radial distance $r$. Since $R_{i t}$ is a Rayleigh distributed random variable, for a given $r, \gamma$ has an exponential distribution.

Figure 4 compares the throughputs of the LIT and LAT controls to the throughput of the protocol when the location information is unavailable. The network size is assumed to be $n=1000$. Since the location information is absent, the channel gain is assumed to be

$$
\gamma_{i}^{(t)}=\frac{R_{i t}^{2}}{h^{2}}
$$

As can be seen from the figure, for the given set of parameters, the throughput of LIT and LAT are almost identical. The difference between the two controls is 


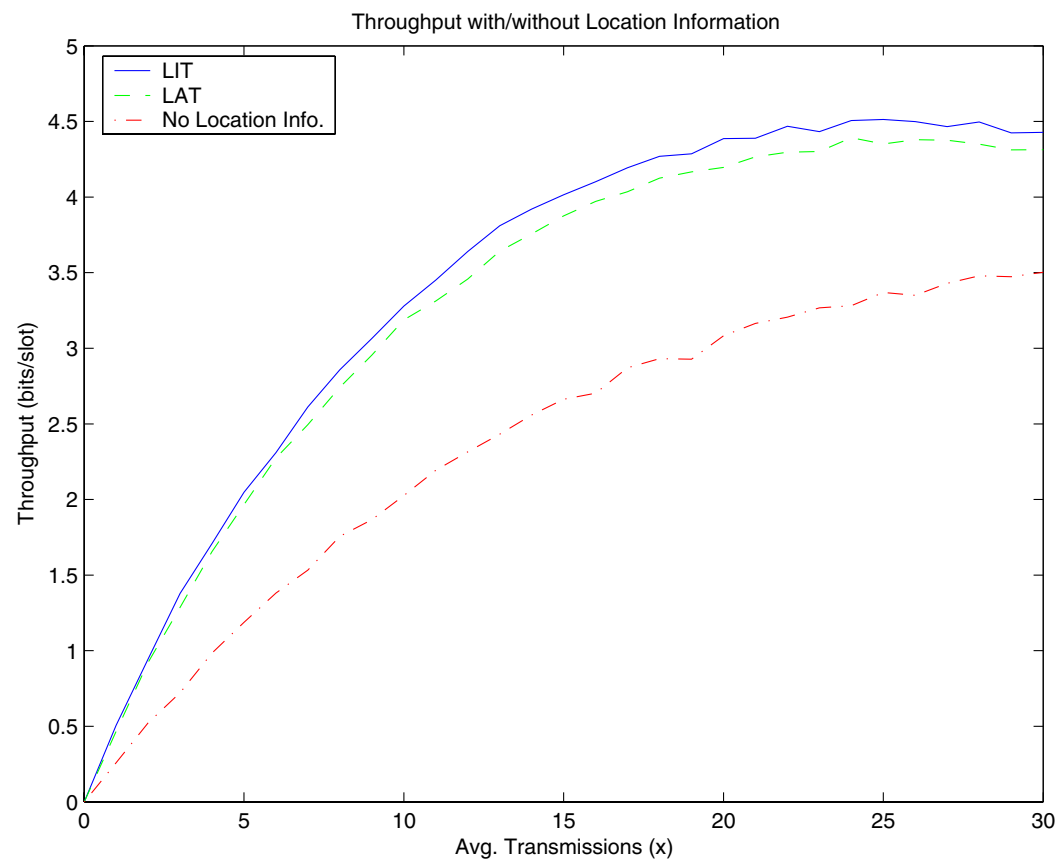

Figure 4. Throughput without location information.

the final distribution of transmitting sensors with respect to location [13]. However, when the location information is not available in the form of measurement or distribution, the throughput is lower than the LAT and LIT controls. The absence of necessary side information results in an incorrect assumption of prior channel state distribution and the pdf of aposteriori CSI does not converge to the desired target distribution.

\section{Robustness and Sensitivity}

In this section, we discuss the performance of the O-ALOHA protocol under errors in assumption of channel state distribution, size and other parameters through simulations. The assumptions of the channel and parameters of the network are as mentioned in Section 4.2. We consider a particular implementation of the O-ALOHA protocol to demonstrate the sensitivity. The parameters of the protocol have not been optimized to obtain maximum throughput.

\subsection{Error in Path-Loss Parameter}

We consider the effect of incorrect path loss parameter on the performance of O-ALOHA. We assume that the measurement of $r$ is available and consider the LAT control. The path loss is inversely proportional to $r^{\alpha}$, where $\alpha$ is a positive constant. The actual value of $\alpha=1.5$. Figure 5 shows the throughputs of the protocol when the assumed $\alpha=1.1$ and $\alpha=2$. It is clear from the figures that the transmission control is highly sensitive to the path loss parameter. Since the underlying channel gain is assumed to be exponentially distributed, a small change in the order of $r$ leads to significant change in throughput. It is also interesting to note that an overestimation of the path loss parameter yields a higher throughput than the accurate protocol. The reasoning behind this is that, when the path-loss parameter is overestimated, the transmission control results in an increase in the number of transmissions, and hence a better throughput for the same value of $x$. Similarly, when $\alpha$ is underestimated, the throughput is lower because for the same $x$, the average number of actual transmissions is much lower than the designed value. Therefore, a good strategy to retain a high throughput would be to design the transmission control with a higher value of $\alpha$ than expected to allow for variations. Such a strategy would however result in an increased number of transmissions than desired and could prove detrimental to the efficiency of the network. 


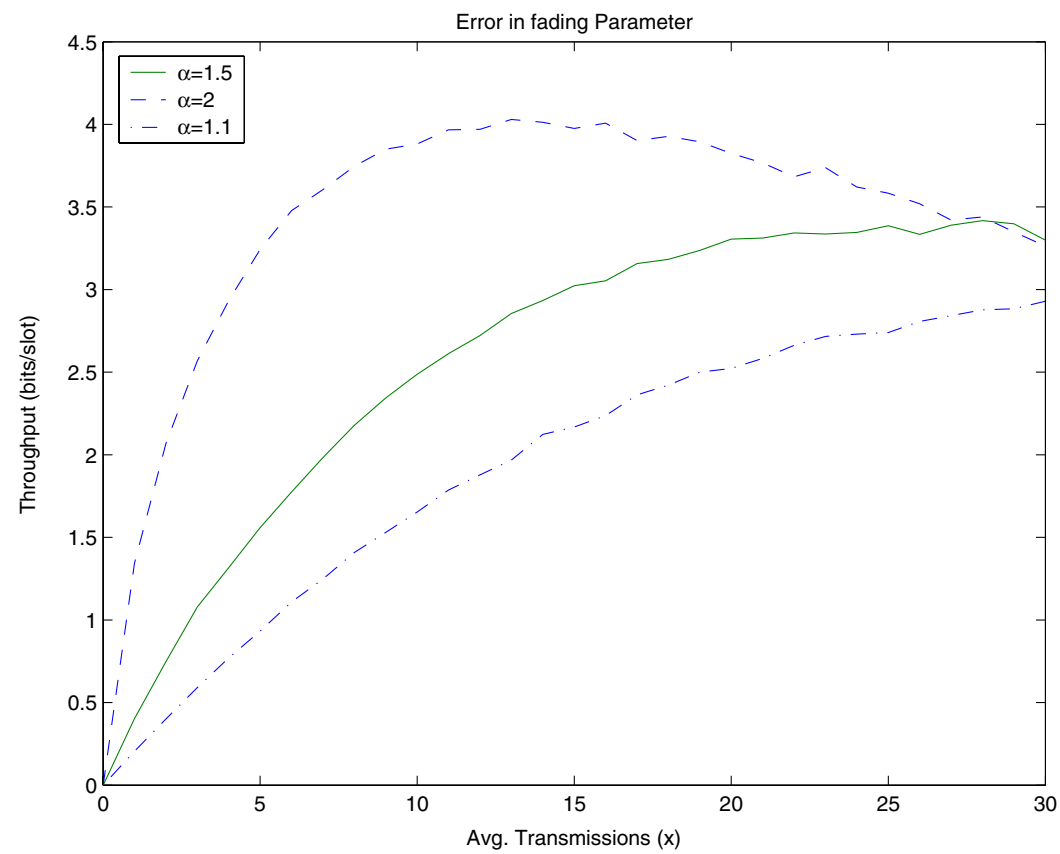

Figure 5. Incorrect path loss.

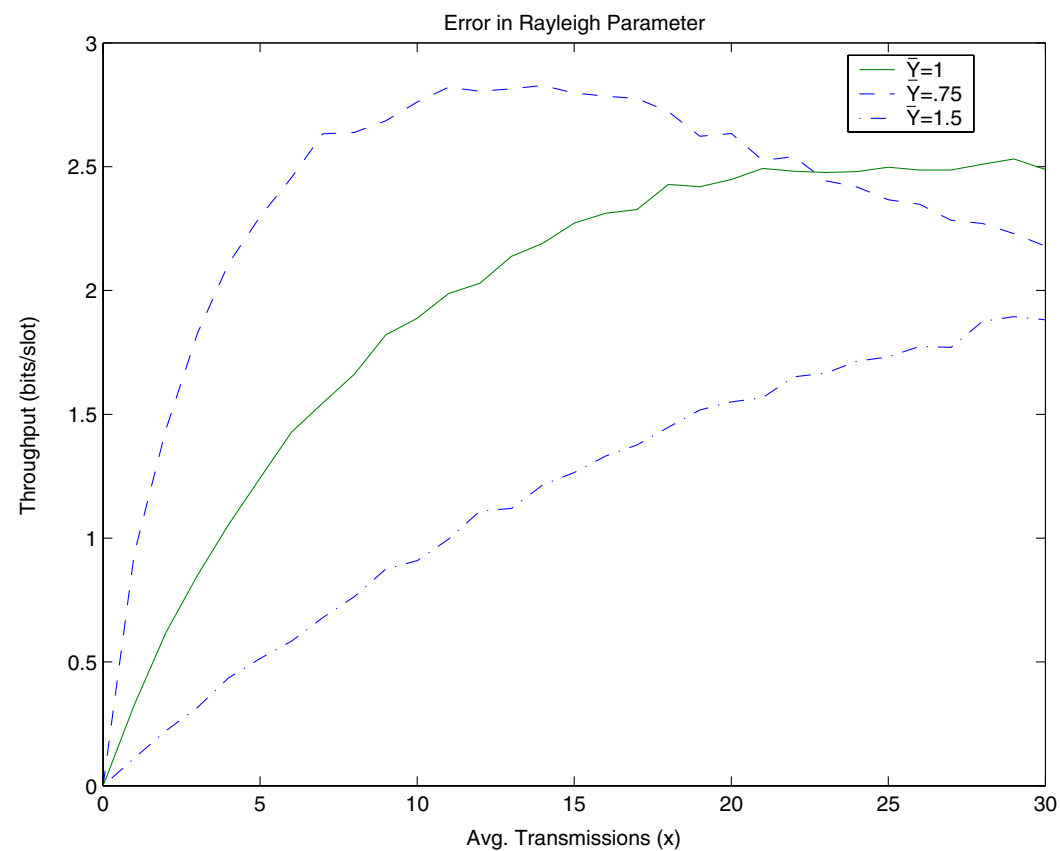

Figure 6. Error in fading parameter.

\subsection{Error in Rayleigh Fading Parameter}

We now consider the performance of the protocol under error in assumption of the Rayleigh fading parameter. As given in (11), the fading depends on $R_{i t}{ }^{2}$, where $R_{i t}$ is Rayleigh distributed. We consider $Y=R_{i t}^{2}$, an exponentially distributed random variable as the fading parameter. For the given network, let $Y$ be distributed with mean $\bar{Y}$. Figure 6 plots the throughput of the LAT control when the assumed $\bar{Y}=1.5$ and $\bar{Y}=.75$ whereas 


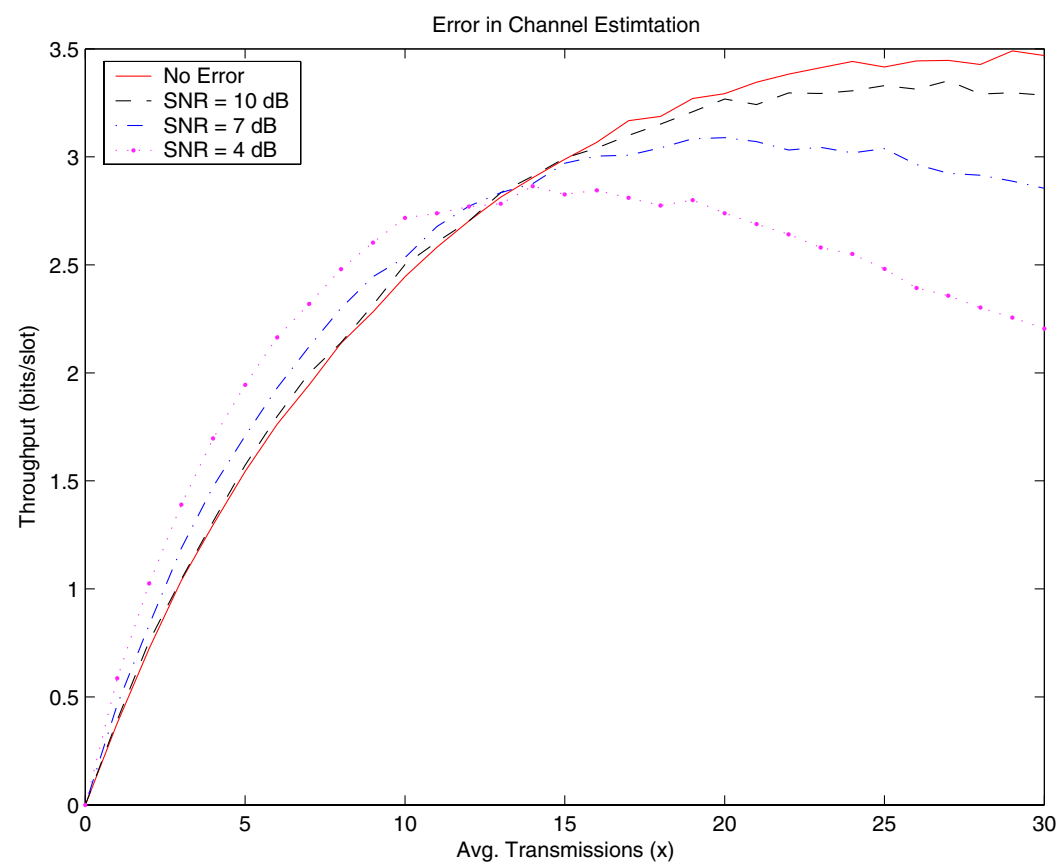

Figure 7. Error in channel estimation.

the actual value is $\bar{Y}=1$. When the Rayleigh parameter is underestimated, the performance is better than the accurate transmission control. An underestimation of the parameter results in a different shape of the assumed CSI distribution. Therefore, for a given value of $x$, the number of transmitters with a good $\gamma$ is higher than the estimated number. This results in an increase in the number of transmissions than the designed $x$, and hence a higher throughput. Similarly, an overestimation results in a reduced average transmissions per slot and hence a lower throughput. The transmission control in this case should assume a lower estimate of the parameter, so that variations in the parameter do not reduce the throughput.

\subsection{Error in Channel Estimation}

In this section, we consider error in the estimation of the channel gain through the beacon. It is assumed that the noise in beacon transmission is AWGN with variance $N_{o}$. If the beacon is assumed to have unit power and the variance of the channel gain can be represented by $\bar{\gamma}$, we can write the average SNR as

$$
S N R=\frac{\bar{\gamma}}{N_{o}} .
$$

Figure 7 plots the throughput for different values of SNR. It can be seen that, a significant change in throughput is observed only for very low values of SNR. This is because only signals with low channel gain are affected significantly by noise and the transmission control ensures that only sensors with high channel gain transmit.

For some target distributions, it is possible to modify the transmission control to account for the additive noise in the channel gain estimate. Let $\gamma^{\prime}$ represent the channel gain estimated by the sensor. Let $f_{e}\left(\gamma^{\prime}\right)$ represent the channel state distribution as seen by the sensors. We know that

$$
\gamma^{\prime}=\gamma+n, \quad \text { where } \quad n \sim w(n) .
$$

Therefore, if $F(\omega), W(\omega)$ and $F_{e}(\omega)$ represent the characteristic functions of $f(\gamma), w(n)$ and $f_{e}\left(\gamma^{\prime}\right)$ respectively, then

$$
F_{e}(\omega)=F(\omega) W(\omega) .
$$

We shall assume the transmission control to be of the form

$$
s_{n}\left(\gamma^{\prime}\right)=q \min \left(\frac{x}{n} \frac{t_{e}\left(\gamma^{\prime}\right)}{f_{e}\left(\gamma^{\prime}\right)}, 1\right) .
$$




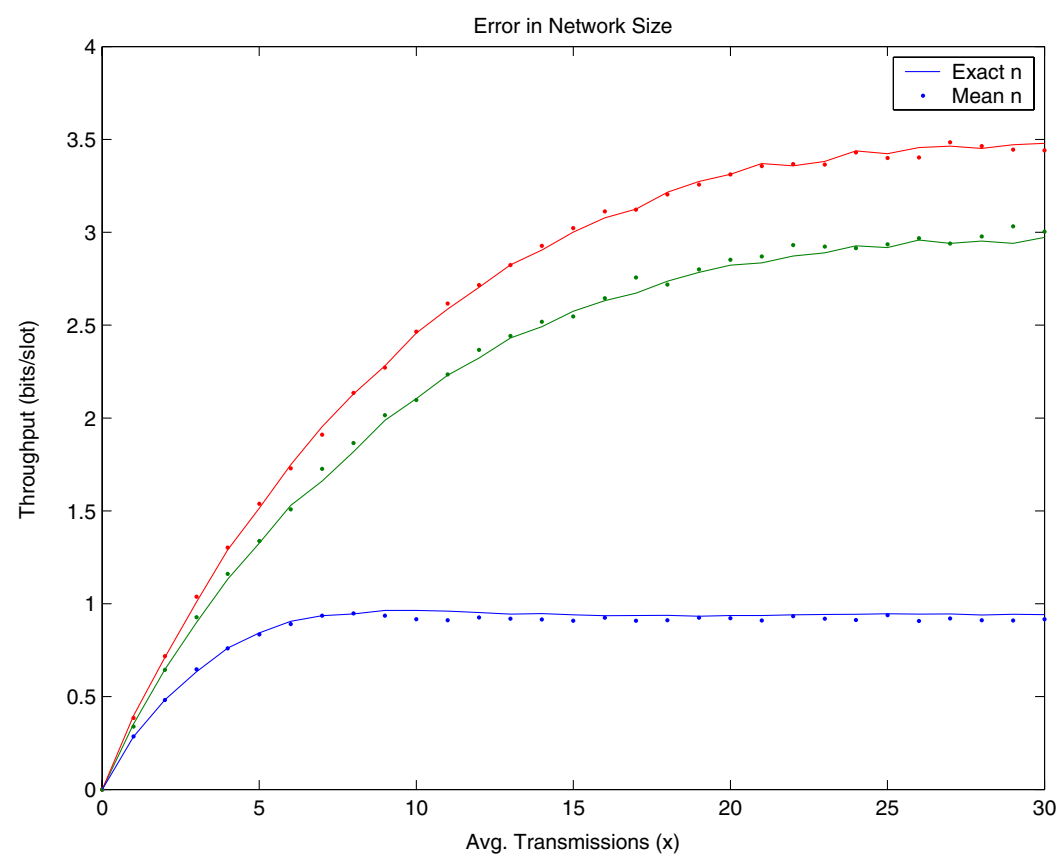

Figure 8. Error in network size.

The transmission control in (15) shapes the aposteriori distribution of the estimated channel state $\gamma^{\prime}$ to the distribution $t_{e}\left(\gamma^{\prime}\right)$. We need to choose an appropriate $t_{e}(\cdot)$ so that the aposteriori distribution of the channel state $\gamma$ observed by the mobile AP is $t(\cdot)$. If we assume the characteristic functions of $t(\gamma), t_{e}\left(\gamma^{\prime}\right)$ to be equal to $T(\omega)$ and $T_{e}(\omega)$ respectively, from (14), we can say that

$$
T_{e}(\omega)=T(\omega) W(\omega)
$$

Therefore, if we can obtain the respective characteristic functions, then we can appropriately design the transmission control based on the estimate $\gamma^{\prime}$ such that the actual channel gain $\gamma$ has an aposteriori distribution equal to $t(\gamma)$. This ensures that the desired asymptotic throughput is achieved.

\subsection{Error in Network Size}

In practical sensor networks, it is not possible to estimate the size accurately. Due to random deployment, the number of sensors in the area activated by the mobile AP may not have a fixed number of sensors. Secondly, the sensors have low duty cycles and are known to temporarily go into SLEEP mode and get activated again later. Therefore, the size of the network is not a constant in every slot. We assume that the number of sensors is distributed as a Poisson random variable. The motivation behind the Poisson assumption is that, the distribution of sensors in a region is dependent only on the area of the region [16]. Figure 8 plots the throughput for networks of different sizes, where the mean of the Poisson distribution is used as the size $n$ in the transmission control. As can be seen from the figure, even for a small $n$, the throughput is almost identical to the throughput where the actual size is known. Thus the system is robust to changes in size.

\section{Coded Random Access}

An interesting feature of sensor networks is collaborative transmission. After the sensing phase, all sensors in the network can collaborate and agree upon the transmission of a particular message. The transmissions can then be coded in order to compensate for packet loss. In this section, we discuss different coding schemes for this purpose. For each of these schemes, we characterize $C$, the number of bits per slot that can be reliably transmitted back to the mobile $\mathrm{AP}$ and $\mathcal{E}$, the random coding error exponent. In all the schemes discussed, it is assumed that if a packet is successfully received, 


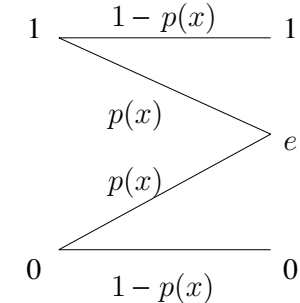

Figure 9. Erasure channel model.

then it is decoded without any error. Therefore, the channel model is a binary erasure channel as shown in Fig. 9.

We first consider two schemes, where the message is coded across time. These schemes have been well discussed in [13]. We then propose a scheme where the message is coded across sensors.

\subsection{Spreading Code Independent Transmission}

We assume that the entire network has agreed upon the transmission of a message $k$ from a common binary codebook of size $\left\lceil 2^{\mathrm{MR}}\right\rceil \times M$, where $M$ is the length of the codeword. In slot $t$, the mobile AP requests the transmission of the $t$ th bit of the codeword through the beacon. All the sensors that decide to transmit in that slot, send the $t^{\text {th }}$ bit in their packet. As shown in [3], the channel between the network and the mobile AP can be viewed as an erasure channel with probability of erasure given by

$$
P_{\text {ind }}(x)=\left(1-\frac{\lambda_{\infty}(x)}{N}\right)^{N}
$$

where $\lambda_{\infty}(x)$ is the asymptotic throughput of the sensor network. The above equation gives the expression for the probability that no packets were successfully received in any of the $N$ orthogonal spreading codes. The achievable rate of the binary erasure channel [15] for Spreading code independent transmission given $x$ is

$$
C_{\text {ind }}(x)=1-\left(1-\frac{\lambda_{\infty}(x)}{N}\right)^{N} \text { bits/slot. }
$$

For a codebook size $\left\lceil 2^{M R}\right\rceil \times M$, the probability of detection error can be upper bounded by error exponent expressions [3]

$$
P_{e}^{\text {ind }}(M, x) \leq \exp \left(-M \log _{e}(2) \mathcal{E}_{\text {ind }}(R, x)\right)
$$

$$
\mathcal{E}_{\text {ind }}(R, x)=\left\{\begin{array}{l}
-(1-R) \log _{2}\left(\frac{P_{\text {ind }}(x)}{1-R}\right) \\
\quad-R \log _{2}\left(\frac{1-P_{\text {ind }}(x)}{R}\right), \\
R<1-P_{\text {ind }}(x) \\
0, \\
R \geq 1-P_{\text {ind }}(x)
\end{array} .\right.
$$

\subsection{Spreading Code Dependent Transmission}

In this section we consider a modified version of the previous scheme, where the bit transmitted is dependent on the spreading code used. It is assumed that the entire network agrees upon the transmission of message $k$ from a common binary codebook of size $\left\lceil 2^{\mathrm{M}^{\prime} \mathrm{NR}}\right\rceil \times M^{\prime} N$, where $M=M^{\prime} N$ is the codeword length. Each codeword is viewed as a two dimensional array of size $M^{\prime} \times N$. The $N$ orthogonal spreading codes are ordered from 1 to $N$. In the $i$ th slot, the mobile AP sends a request for transmission of the $i$ th row of the codeword, through the beacon. All the sensors that decide to transmit using the $j$ th spreading code, transmit bit $(i, j)$ of the chosen codeword. The probability of erasure in this case is the probability that no packet was received successfully on a particular spreading code which is given by

$$
P_{\text {dep }}(x)=1-\frac{\lambda_{\infty}(x)}{N}
$$

It has been shown that the transmission of each bit is i.i.d. Therefore, the achievable rate in bits/channel use of the Spreading code dependent transmission is $\frac{\lambda_{\infty}(x)}{N}$. Since there are $N$ channel uses per slot, the achievable rate given $x$, in bits per slot is

$$
C_{\text {dep }}(x)=\lambda_{\infty}(x) \text { bits/slot }
$$

and the error exponent is given by

$$
\mathcal{E}_{\mathrm{dep}}(R, x)=\left\{\begin{array}{r}
-(1-R) \log _{2}\left(\frac{P_{\mathrm{dep}}(x)}{1-R}\right) \\
\quad-R \log _{2}\left(\frac{1-P_{\mathrm{dep}}(x)}{R}\right), \\
R<1-P_{\mathrm{dep}}(x) \\
0, \\
R \geq 1-P_{\mathrm{dep}}(x)
\end{array} .\right.
$$




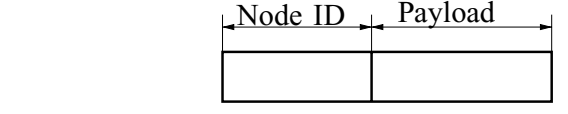

Figure 10. Packet structure.

In the two schemes discussed, it is necessary for the mobile AP to send information through the beacon, which is decoded by the sensors. This demands more processing power on part of the sensors. We next propose a scheme, where the beacon does not contain any information, and the sensors use an energy detector to estimate the channel gain from the beacon.

\subsection{Coding Across Sensors}

In this scheme, the message to be transmitted to the mobile AP is encoded across sensors, and each sensor is required to transmit its ID along with the data. We assume that the packets transmitted by the sensors each have the structure as shown in Fig. 10. The node ID typically has a length $\log n$, where $n$ is the size of the network.

The sensor network is assumed to employ a binary code book of size $\left\lceil 2^{\mathrm{nR}}\right\rceil \times n$ to transmit data, where $n$ is the number of sensors. If the sensors decide upon the transmission of message $k$, where $k \in\left\{1, \ldots,\left\lceil 2^{n R}\right\rceil\right\}$, to the mobile AP, the encoding is performed as follows. The payload of sensor $i$ contains the $i$ th bit of the codeword $m$. We assume that the mobile AP stays over the sensor network for $\rho n$ slots ( $\rho$ is a design parameter). During this time, each sensor repeats the same bit in each slot. Since, the throughput of the reachback protocol is $\lambda\left(n, s_{\mathrm{n}}(\cdot)\right)$ and the packet from sensor $i$ is not received by the mobile AP only if it is unsuccessful in every one of the $\rho n$ slots, the channel between each sensor and the mobile AP can be modeled as an erasure channel with erasure probability

$$
P_{\text {sen }}=\left(1-\frac{\lambda\left(n, s_{n}(\cdot)\right)}{n}\right)^{\rho n} \text {. }
$$

This is shown in Fig. 9. Strictly speaking, in order to use the information theoretic results from the literature on discrete memoryless channels (DMC), we need to show that successful reception of different sensors is independent. It is however difficult to show that this is true, since the sensors interfere with one another. For the moment we ignore the fact that the receptions are correlated and assume that we can model the transmissions from the sensors as a DMC. Therefore reliable

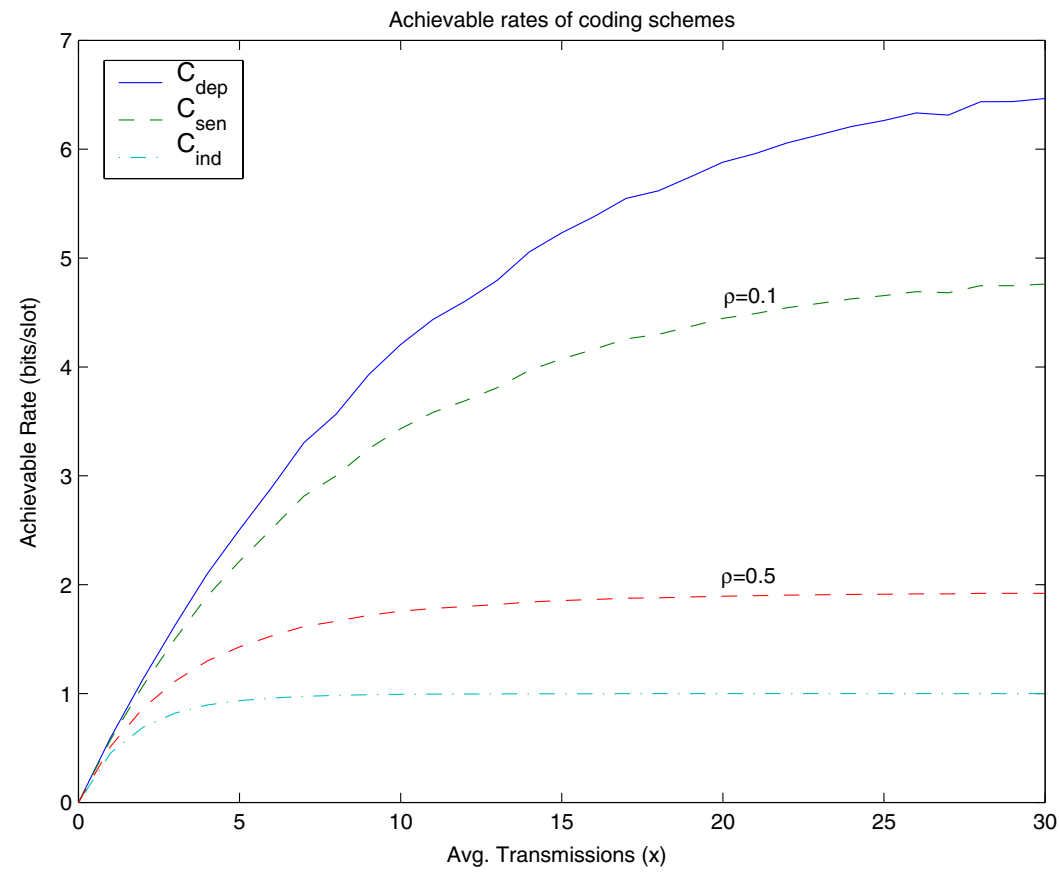

Figure 11. Comparison of achievable rates. 
transmission at $C$ bits/slot is possible if

$$
C \leq \frac{1-P_{\text {sen }}}{\rho}
$$

The probability of success of a particular sensor is $1-$ $p(n)$. Since $n$ sensors attempt to transmit every slot and the mobile AP performs the collection for $\rho n$ slots, we get the above equation for the achievable rate of the system. As $n$ becomes larger, $\lambda\left(n, s_{n}(\cdot)\right) \rightarrow \lambda_{\infty}(x)$, and the erasure probability converges

$$
p(n) \rightarrow e^{-\lambda_{\infty}(x) \rho} \triangleq p(x, \rho)
$$

Hence for $n$ large, the achievable rate of the system is given by

$$
C_{\text {sen }}=\frac{1-e^{-\lambda_{\infty}(x) \rho}}{\rho} \text { bits } / \text { slot. }
$$

It is also interesting to characterize how the detection probability varies with the number of sensors $n$, rate $R$ and dwell time $\rho$. The detection probability can be bounded by the method of error exponents and we have for large $n$

$$
P_{e}(n, R, x, \rho) \leq \exp \left(-n \log _{e}(2) \mathcal{E}_{\text {sen }}(R, x, \rho)\right),
$$

where $\mathcal{E}(R, x, \rho)=0$ for $R \geq 1-p(x, \rho)$ and for $R<1-p(x, \rho)$,

$$
\begin{aligned}
\mathcal{E}_{\text {sen }}(R, x, \rho)= & -(1-R) \log _{2}\left(\frac{p(x, \rho)}{1-R}\right) \\
& -R \log _{2}\left(\frac{1-p(x, \rho)}{R}\right)
\end{aligned}
$$

\section{Comparison of Coding Schemes}

In this section, we shall compare the different coding schemes discussed using an implementation of the O-ALOHA protocol. The parameters of the transmission control are identical to the example discussed in Section 4.2.

\subsection{Achievable Rates}

Figure 11 plots the achievable rates of the different coding schemes versus the design parameter $x$, the average transmissions per slot. The spreading code dependent scheme has the best achievable rates, equal to the throughput of the protocol. The spreading code independent scheme has a low achievable rate

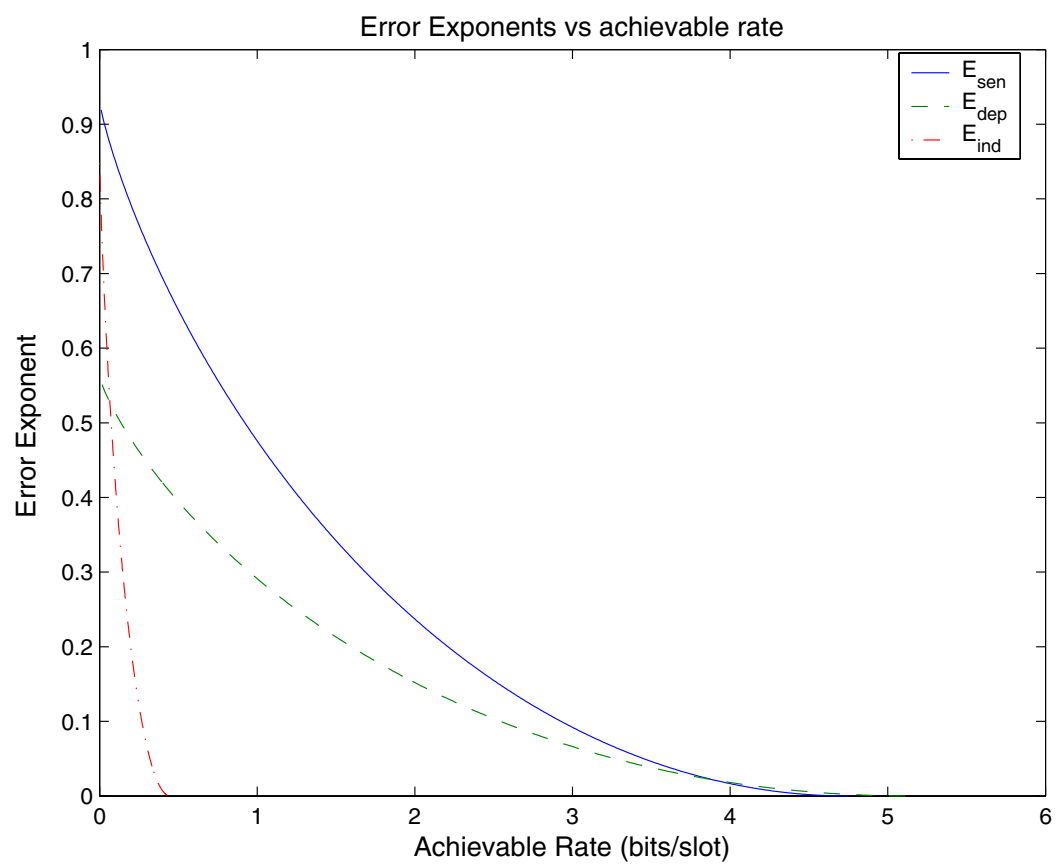

Figure 12. Comparison of error exponents. 

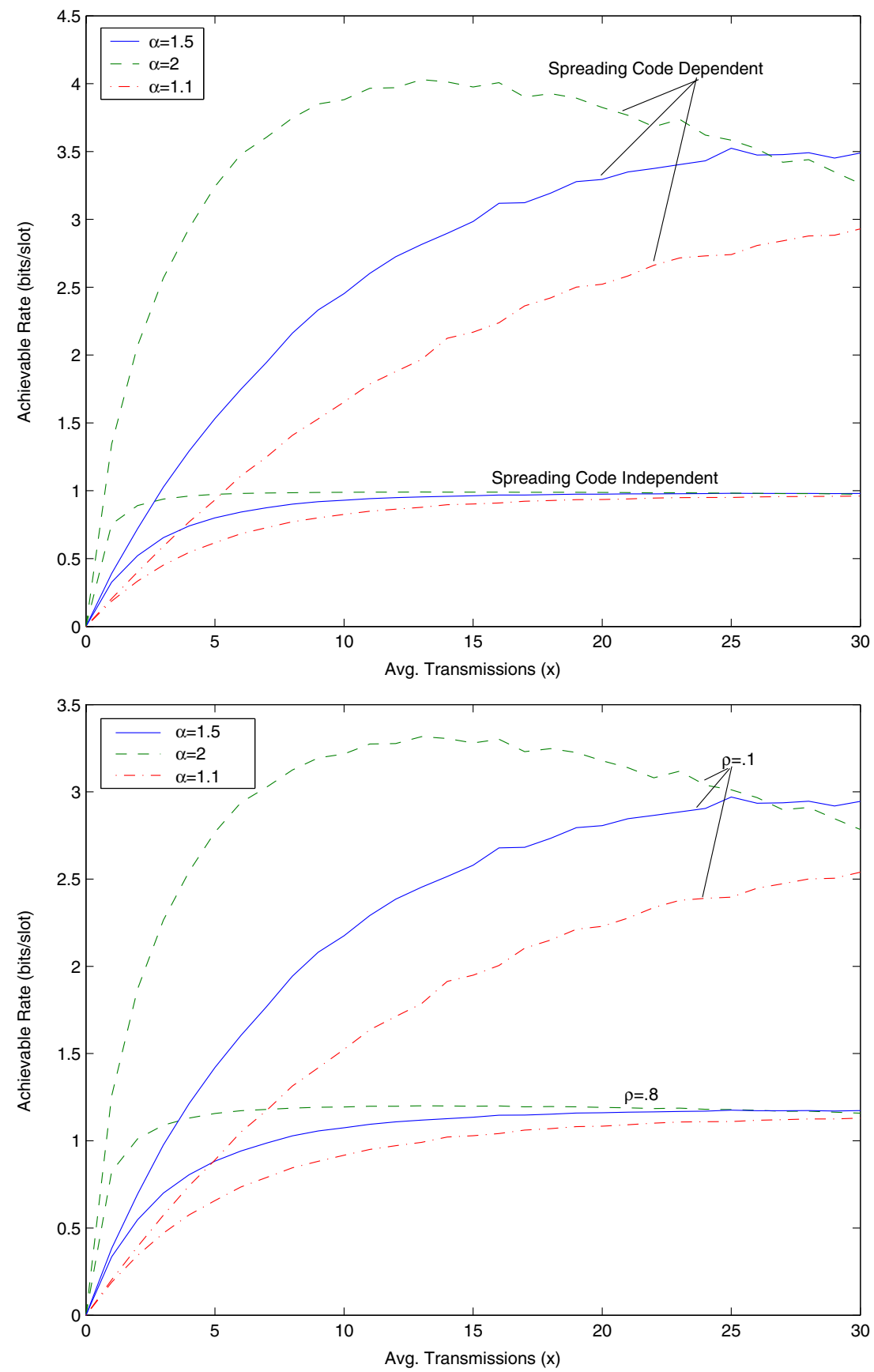

Figure 13. Sensitivity of coding schemes.

compared to other coding schemes. This is because of the in-built redundancy in the form of repetitive coding within a slot, which in some cases yields a better error exponent (see Section 7.2). The achievable rate of the coding over sensors scheme increases monotonically towards the throughput with decrease in $\rho$. This is clear from the fact that

$$
\lim _{\rho \rightarrow 0} \frac{1-e^{-\rho \lambda_{\infty}(x)}}{\rho}=\lambda_{\infty}(x) .
$$

The coding over sensors scheme requires the transmission of the sensor ID unlike the schemes involving 
coded random access. This is an additional overhead in every packet transmitted. However, in the other two schemes, the mobile AP is required to transmit data through the beacon. The sensors, in addition to estimating channel gain, need to decode data from the beacon, therefore require more processing power.

\subsection{Random Coding Error Exponents}

As discussed in the previous section, for each coding scheme, the probability of detection error can be bounded using the method of error exponents. In order to compare the schemes, it is necessary to consider a common codebook and equal energy for the transmission of a bit.

Figure 12 compares the error exponents of the proposed coding schemes. It is clear from the figure that the achievable rate for the coding across sensors scheme and the spreading code dependent scheme are much higher in comparison to the spreading code independent scheme. However for low rate codebooks, the spreading code independent scheme has a better error probability. This can be attributed to the fact that this scheme has an inherent repetitive code that adds to the redundancy. The spreading code dependent and the coding across sensors scheme have comparable achievable rates but the coding across sensors has a better error probability. This scheme again has an inbuilt repetitive code, wherein each sensor transmits the same bit every slot. The parameters in the plot are chosen such that the average transmissions per bit of the codeword are equal for the three schemes in order to provide a fair comparison.

\subsection{Sensitivity of Coding Schemes}

Figure 13 demonstrates the effect of coded random access on sensitivity. As seen in Section 5, the protocol is sensitive to parameters of the channel state distribution. However, this sensitivity can be overcome using some of the coding schemes discussed previously. Figure 13 plots the achievable rates of the protocol when there is a change in the path-loss parameter. Since the rate for the spreading code dependent scheme is directly proportional to the throughput, it is sensitive to the change in parameter. However, as can be seen from the figure, the spreading code independent scheme normalizes the variation in throughput. This is because the embedded repetitive coding involved in the scheme reduces the sensitivity of the protocol to change in parameter.
Figure 13 also plots the variation in achievable rates with change in path-loss parameter for the coding over sensors scheme. The sensitivity of the achievable rate to the change in parameter reduces with the increase in $\rho$. This is because, in this scheme, the sensors attempt to transmit the same bit in every slot, and a higher $\rho$ implies more number of slots for transmission and therefore, more redundancy.

\section{Conclusion}

In this work, we consider sensitivity and coding issues of a distributed random access protocol, Opportunistic ALOHA, for reachback in sensor networks with mobile access points (AP). With throughput as the metric, we demonstrate the gain in using sensor location information as a part of the transmission control, either in the form of probability distribution or as a measurement. We found that the protocol was robust to errors in network size and estimated channel gain, whereas it was sensitive to fading and path loss parameters.

We proposed three coding schemes for reliable transmission. For the spreading code independent and spreading code dependent schemes, the coding was done across time and required data to be transmitted through the beacon and decoded by the sensors. Whereas the coding across sensors scheme mandated the transmission of node ID in every packet. These schemes combine coding with the random access and can be used in combination with low density parity check codes or turbo codes to achieve high code rates. In this paper, we have assumed that all sensors collaborate and decide upon a single message for transmission. An information theoretic approach to the problem of some sensors having the wrong message has been dealt with in [5].

\section{Acknowledgments}

This work was supported in part by the Multidisciplinary University Research Initiative (MURI) under the Office of Naval Research Contract N0001400-1-0564, Army Research Laboratory CTA on Communication and Networks under Grant DAAD1901-2-0011, and the Army Research Office under Grant ARO-DAAB19-00-1-0507. Part of this work is presented at IEEE Military Communications Conference MILCOM'03, Boston, MA, Oct. 13-16, 2003. 


\section{Note}

1. Submitted to Special Issue on MMSP02 of the JVSPS , July 2004

\section{References}

1. T. Rappaport, Wireless Communications Principles and Practice, Prentice Hall, 1996.

2. T. Cover and J. Thomas, Elements of Information Theory, John Wiley \& Sons, Inc., 1991.

3. Robert G. Gallager, Information Theory and Reliable Communication, John Wiley and Sons, Inc., New York, NY, 1968.

4. A. Chandra, V. Gummalla, and J.Limb, "Wireless Medium Access Protocols," IEEE Communications Surveys, Second Quarter, 2000.

5. Z. Yang and L. Tong, "Cooperative Sensor Networks with Misinformed Nodes," to appear in IEEE Transactions on Information Theory, Dec. 2005.

6. B. Hajek, A. Krishna, and R.O. LaMaire, "On the Capture Probability for a Large Number of Stations," IEEE Trans. Communications, vol. 45, 1997, pp. 254-260.

7. J.F. Akyildiz, W. Su, Y. Sankarasubramaniam, and E. Cayirci, "A Survey on Sensor Networks," in IEEE Communications Magzine, vol. 40, 2002, pp. 102-114.

8. M. Dong, L. Tong, and B. Sadler, "Impact of MAC Design on Signal Field Reconstruction in Dense Sensor Networks," submitted to IEEE Trans. on Signal Processing, Nov. 2003.

9. Y. Sung and L. Tong, "Asymptotic Locally Optimal Detector for Large Scale Sensor Networks under the Poisson Regime," Accepted to IEEE Trans. Signal Processing, Feb. 2004.

10. S. Adireddy and L. Tong, "Exploiting Decentralized Channel State Information for Random Access," IEEE Transactions on Information Theory, vol. 51, no. 2, pp. 537-561, Feb. 2005.

11. M. Grossglauser and D. Tse, "Mobility Increases the Capacity of Wireless Adhoc Networks," in IEEE/ACM Trans. Networking, vol. 10, 2002, pp. 477-486.

12. K. Sohrabi, J. Gao, V. Ailawadhi, and G. Pottie, "Protocols for Self-Organization of a Wireless Sensor Network," IEEE Personal Communication, pp. 16-27, October, 2000.

13. P. Venkitasubramaniam, S. Adireddy, and L. Tong, "Sensor Networks with Mobile Access: Optimal Random Access and Coding," IEEE Journal on Selected Areas in Communications: Special Issue on Sensor Networks, vol. 22, no. 6, pp. 1058-1068, August 2004

14. G. Mergen, Q. Zhao, and L.Tong, "Sensor Networks with Mobile Access: Energy and Capacity Considerations," submitted to IEEE Transactions on Communications, revised in June 2005.

15. W. Zhao and M.H. Ammar, "Message Ferrying: Proactive Routing in Highly-Partitioned Wireless ad hoc Networks," in Proc. 9th IEEE Workshop on Future Trends of Distr. Computing Systems, 2003, 308-314.

16. P. Venkitasubramaniam and L. Tong, "Optimal Configurations of Mobile Access Points for Sensor Data Collection," ACSP Tech. Report ACSP-TR-11-04-02, Cornell University, Ithaca, NY.
17. P. Venkitasubramaniam, Q. Zhao and L. Tong, "Sensor Networks with Multiple Mobile Access Points," in Proc. 2004 CISS, Princeton, NJ, March 2004.

18. Q. Zhao and L. Tong, "Distributed Opportunistic Transmission for Wireless Sensor Networks," ICASSP 2004, Montreal, CA, May 2004.

19. M. Dong, L. Tong, and B.M. Sadler, "Effect of MAC Design on Source Estimation in Dense Sensor Networks," in ICASSP 2004, Montreal, CA, May 2004.

20. Y. Sung, L. Tong, and A. Swami, "Asymptotic Locally Optimal Detector in Large Scale Sensor Networks under the Poisson Regime," ICASSP, Montreal, Canada, May 2004.

21. S. Adireddy and L. Tong, "Medium Access Control using Channel State Information for Large Sensor Networks," in Proc. of the IEEE Workshop on Multimedia Signal Processing 2002, St. Thomas, US Virgin Islands, Dec. 2002, http://acsp.ece.cornell.edu/pubC.html/\}.

22. W.J. Kaiser, G.J. Pottie, M. Srivastava, G.S. Sukhatme, J. Villasenor, and D. Estrin, "Networked Infomechanical Systems (NIMS) for Ambient Intelligence," CENS Technical Report, Dec. 2003.

23. N. Bansal and Z. Liu, "Capacity, Delay and Mobility in Wireless ad-hoc Networks," in Proc. of 2003 INFOCOM, San Francisco, CA, March 30-April 32003.

24. W. Zhao and M.H. Ammar, "Message Ferrying: Proactive Routing in Highly-Partitioned Wireless ad hoc Networks," in Proc. of the IEEE Workshop on Future Trends in Distributed Computing Systems, Puerto Rico, May 2003.

25. L. Tong, Q. Zhao, and S. Adireddy, "Sensor Networks with Mobile Agents," in Proc. 2003 Military Communications Intl Symp, Boston, MA, Oct. 2003.

26. R.C. Shah, S. Roy, S. Jain, and W. Brunette, "Data MULEs: Modeling a Three-tier Architecture for Sparse Sensor Networks," in IEEE Workshop on Sensor Network Protocols and Applications (SNPA), May 2003.

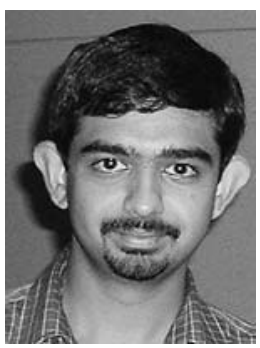

Parvathinathan Venkitasubramaniam was born in India in 1981. $\mathrm{He}$ received his B.Tech. degree from the department of Electrical Engineering, Indian Institute of Technology, Madras in 2002. He joined the School of Electrical and Computer Engineering, Cornell University, Ithaca, NY, in 2002 and he is working toward his Ph.D. degree. He is a recipient of the 2004 Leonard G. Abraham Award (with S. Adireddy and L. Tong) from the IEEE Communications Society. His research interests include random-access protocols,sensor networks, and information theory.

pv45@cornell.edu 


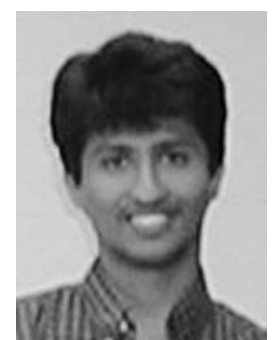

Srihari Adireddy was born in India in 1977. He received the B.Tech. degree from the Department of Electrical Engineering, Indian Institute of Technology, Madras, and M.S. and Ph. D. degrees from the School of Electrical and Computer Engineering, Cornell University, Ithaca, NY in 2001 and 2003 respectively. Currently, he is working at Silicon Laboratories, Austin, TX. He is a recipient of the 2004 Leonard G. Abraham Award (with P. Venkitasubramaniam and L. Tong) from the IEEE Communications Society. His research interests include signal processing, information theory, and randomaccess protocols.

srihari@ece.cornell.edu

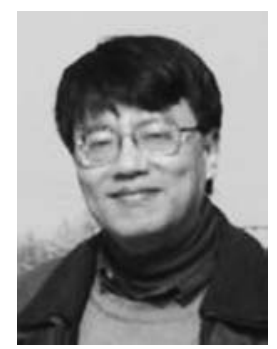

Lang Tong received the B.E. degree from Tsinghua University, Beijing, China, in 1985, and M.S. and Ph.D. degrees in electrical engineering in 1987 and 1990, respectively, from the University of Notre Dame, Notre Dame, Indiana. He was a Postdoctoral Research Affiliate at the Information Systems Laboratory, Stanford University in 1991. Currently, he is a Professor in the School of Electrical and Computer Engineering, Cornell University, Ithaca, New York.

Dr. Tong received Young Investigator Award rom the Office of Naval Research in 1996, and the Outstanding Young Author Award from the IEEE Circuits and Systems Society in 1991, the 2004 IEEE Signal Processing Society Best Paper Award (with M. Dong), the 2004 Leonard G. Abraham Prize Paper Award from the IEEE Communications Society (with P. Venkitasubramaniam and S. Adireddy). His areas of interest include statistical signal processing, adaptive receiver design for communication systems, signal processing for communication networks, and information theory.

ltong@ece.cornell.edu 